\title{
FREQUENCY INDEPENDENT SELF COMPLEMENTARY BOW TIE ANTENNA DESIGN FOR GPR APPLICATIONS
}

\author{
Saeid KARAMZADEH ${ }^{1, *}$, Oğuz Furkan KILIÇ ${ }^{1}$, Fatih DEMİRBAŞ ${ }^{1}$, Ahmet Said HEPBİÇER ${ }^{1}$ \\ ${ }^{1}$ Department of Electrical and Electronics Engineering, İstanbul Aydın University, İstanbul, Turkey
}

\begin{abstract}
Today, the technological evolvements have contributed to the development of Ground Penetration Radar (GPR) systems. GPR systems are used in a lot of fields such as; military field, civilian life and commercial activities. Besides, antenna design for the GPR system is so important. The designed GPR antennas should have lower than $1 \mathrm{GHz}$ start frequency for more penetration and getting information about the object in the deeper layers of ground or any obstacle. Also, with wide band properties obtaining high resolution data would be available. In this paper, a Self-Complementary bow tie antenna is proposed. The start frequency of designed antenna is from $0.45 \mathrm{GHz}$ and it works frequency independently. Simulation and fabrication results are presented for designed antenna.
\end{abstract}

Keywords: GPR, BOW TIE Antenna, Self complementary

\section{INTRODUCTION}

Thanks to improvements in radar systems have been easier to get information about away objects. In here, radar is a system that permits the realization of this happening. Using the radar systems, it is allowing to get more detailed information about an object. At first times radar systems are used with radio waves. High Infrared and Microwave systems use the electromagnetic waves recently [1].

GPR is the special type of radar that with this technology, detecting and tracking buried objects are possible [2]. Civil and military fields are the very popular areas for the Ground Penetration Radar (GPR) applications. In military area, underground warehouses, unexploded bombs, enemy communication channels, bomb shelters and also secret rooms are can be found with GPR systems [3]. In civil life, these systems are used for finding undetected blanks and buried. Also with GPR systems, human who are under the collapsed building are can be determined [4-6].

Generally, two antennas are used for GPR systems. One of them as a receiver and the other one as a transmitter antenna. But some systems use only one antenna as transmitter and receiver at the same time and that called monostatic radar. Electromagnetic waves spread out from the transmitter antenna. Wave's spreading speed is changeable according to medium's permittivity. If the waves hit an object in the medium, some part of waves are reflected from this object Reflected waves are caught by the receiver antenna and analyzed by the system.

The other important property for GPR systems is resolution range of the antenna. More detailed information of objects where in deep could be available with this property.

Physical structure of antenna is also important part of the GPR systems. If the antenna size is small, it provides advantages in use. But, because it is difficult to carry, heavy and large antenna size is not preferable. 
Antenna efficiency is the other important thing. If the antenna gain is high, the information about the object is more detailed and reliable. Antenna gain is affected positively by the physical improvements of antenna, such as size changing or applying of different methods [7].

Many types of antennas are used in the GPR systems. Microstrip antennas, Vivaldi antenna, and horn antennas are some examples of these [8]. To reach greater distance and pass more layers through, the use of circular polarized antenna is advised in the literature [9-11]. Also frequency independent antennas are useful for GPR applications. Frequency independent means that, the bandwidth to as great as 40:1 or more. Frequency independent antennas are principally used in the 10-10,000 $\mathrm{MHz}$ region. These antennas are used in point-to-point communication, television, feeds for reflectors and lenses, and so forth [12].

The self-complementary antenna has proved a broad impedance bandwidth and firstly proposed in 1992 [13-14]. The basic feature of the self-complementary is shown in Figure 1. In theory, a selfcomplementary antenna has a $188.5 \Omega$ input impedance and it doesn't depend on the antenna configuration and source frequency. If the antenna presents itself complementary feature, then it can be shown the frequency-independent features [15].

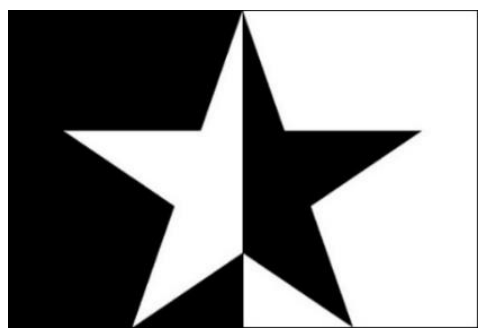

Figure 1. The basic structure of the self-complementary principle

A different method which is self-complementary method was applied to the classic shape of the BowTie antenna. The purpose is to design a more efficient and frequency-independent antenna for GPR systems. The best suitable antenna type is Bow-Tie antenna for GPR systems. It is the most suitable type of antenna in terms of weight and size. In this paper, the results of the self-complementary method implemented on the classic Bow-Tie antenna will be presented. The proposed self-complementary BowTie antenna iterations is simulated in Ansoft HFSS ver.15 computer simulation program. Also the results will be presented. In the last part of this paper, printed self-complementary bow-tie antenna's results will be presented.

\section{GEOMETRY OF ANTENNA}

Geometry of the proposed antenna structure is shown in Figure 2. Epoxy material FR4 substrate whose permittivity is $\varepsilon_{\mathrm{r}}=4.4$, is used for the proposed antenna. Its dimensions are $240 \times 240 \times 1.6 \mathrm{~mm}^{3}$. For impedance matching on the feed side, there is $180 \Omega-50 \Omega$ impedance equalizer circuit just before the antenna feed. Feed path length of antenna $h=150 \mathrm{~mm}$. $L x=110 \mathrm{~mm}$ and $L y=72.62 \mathrm{~mm}$, they affect the antenna operating frequency and efficiency directly. Applied as a result of different designs, the best suitable angles $\theta$ and $\alpha$ are defined as $\theta=71^{\circ}$ and $\alpha=38^{\circ}$.

\section{RESULTS AND DISCUSSIONS}

All parameters are examined and according to the analysis results, optimal diameters of the proposed antenna are chosen. These results are optimized one by one with Ansoft HFSS ver.15. Improvements to the antenna was made in accordance with $\mathrm{s} 11$ (reflection coefficient that should be less than-10dB) graphic results. Development steps were continued as follows. 


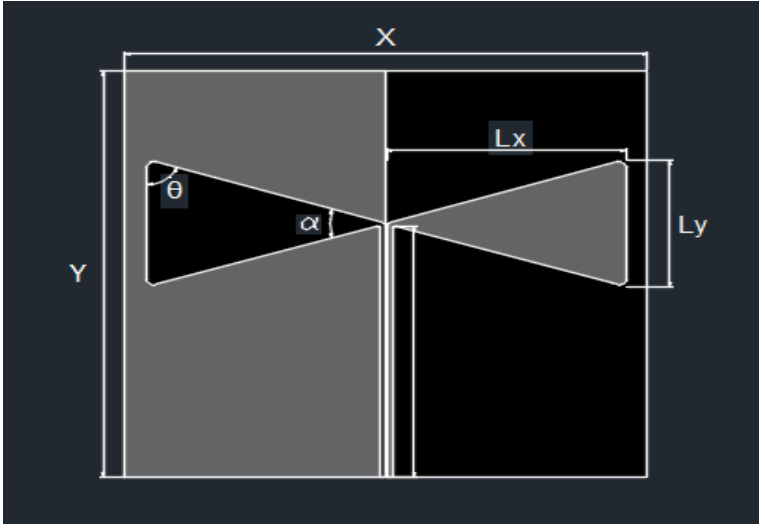

(a)

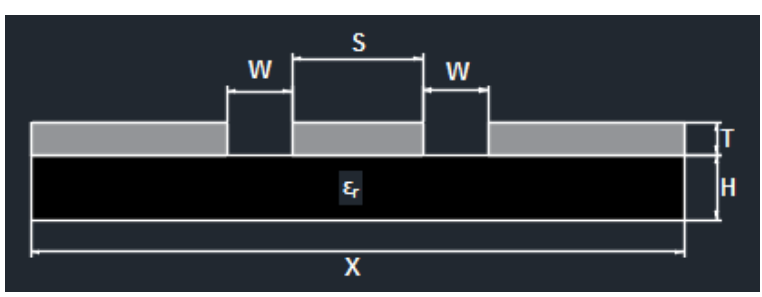

(b)

Figure 2. The geometric feature of the proposed antenna. (a) Top view. (b) Side view

Table I. Antenna Dimensions

\begin{tabular}{|c|c|c|c|c|c|c|}
\hline Antenna & I & II & III & IV & V & VI \\
\hline $\mathbf{x}$ & 240 & 240 & 240 & 240 & 240 & 240 \\
\hline $\mathbf{y}$ & 240 & 240 & 240 & 240 & 240 & 240 \\
\hline $\mathbf{L x}$ & 90 & 90 & 90 & 100 & 105 & 110 \\
\hline $\mathbf{L y}$ & 90 & 86.29 & 76.77 & 74.46 & 72.62 & 72.62 \\
\hline $\boldsymbol{\alpha}$ & 53 & 53 & 48 & 41 & 39 & 38 \\
\hline $\boldsymbol{\theta}$ & 63 & 63 & 66 & 69 & 70 & 71 \\
\hline $\mathbf{h}$ & 150 & 150 & 150 & 150 & 150 & 150 \\
\hline
\end{tabular}

Table I demonstrates the dimension of antenna optimization while designing process one by one. Developing steps are illustrated in the Table I. For all iterations X and Y lengths are equal and 240 millimeters. Lx and Ly values were changed for every antenna design.

By checking the S11 results of antenna, from first form to last form, increasing of the Lx value and decreasing of the Ly value affect the antenna positively. And in the last form $\mathrm{Lx}=110 \mathrm{~mm}$ and $\mathrm{Ly}=72.62$ $\mathrm{mm}$. Also shape of the antenna affects the antenna's gain and s11 value. According to increasing of $\mathrm{Lx}$ and decreasing of Ly, $\alpha$ and $\theta$ values are changed automatically. At the first design $\alpha$ was $53^{\circ}$ and $\theta$ was $63^{\circ}$. At the last form of antenna $\alpha$ is $38^{\circ}$ and $\theta$ was $71^{\circ}$. "h" is the antenna feed way and for all designs $\mathrm{h}=150 \mathrm{~mm}$. To be more uniform current distribution on the antenna, the corners of the triangular structure are smoothed $3 \mathrm{~mm}$. For all designs, $0.8 \mathrm{~mm}, 1.0 \mathrm{~mm}$ and $1.6 \mathrm{~mm}$ FR4 substrate thickness were simulated and compared. Consequently, using $1.6 \mathrm{~mm}$ FR4 substrate has made the best point for s11 values. The Figure 3 demonstrates the all antenna designs' simulation results of s 11 graph according to thickness. In here the best thickness from available form of FR4 for fabrication $(0.8,1$ and $1.6 \mathrm{~mm})$ has been chosen. Each new modification, the antenna has improved slightly. At the first antenna, frequency starts from $1 \mathrm{GHz}$ around and after the optimization steps, starting frequency is coming to $0.45 \mathrm{GHz}$ at last design and desired antenna result is obtained. 
Karamzadeh et al. / Anadolu Univ. J. of Sci. and Technology A-Appl. Sci. and Eng. 18 (1) - 2017

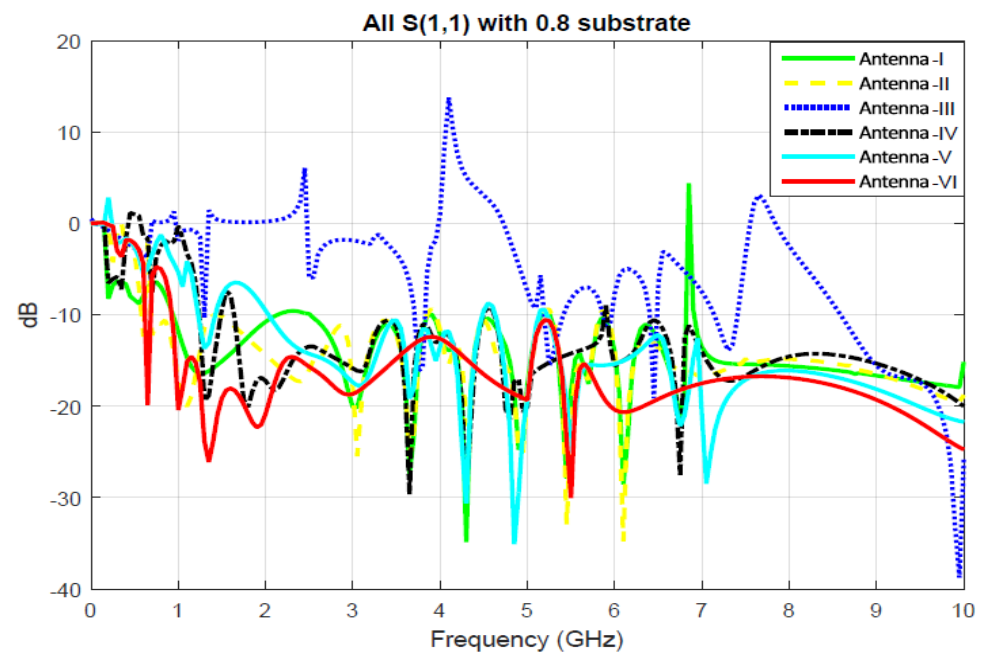

(a)

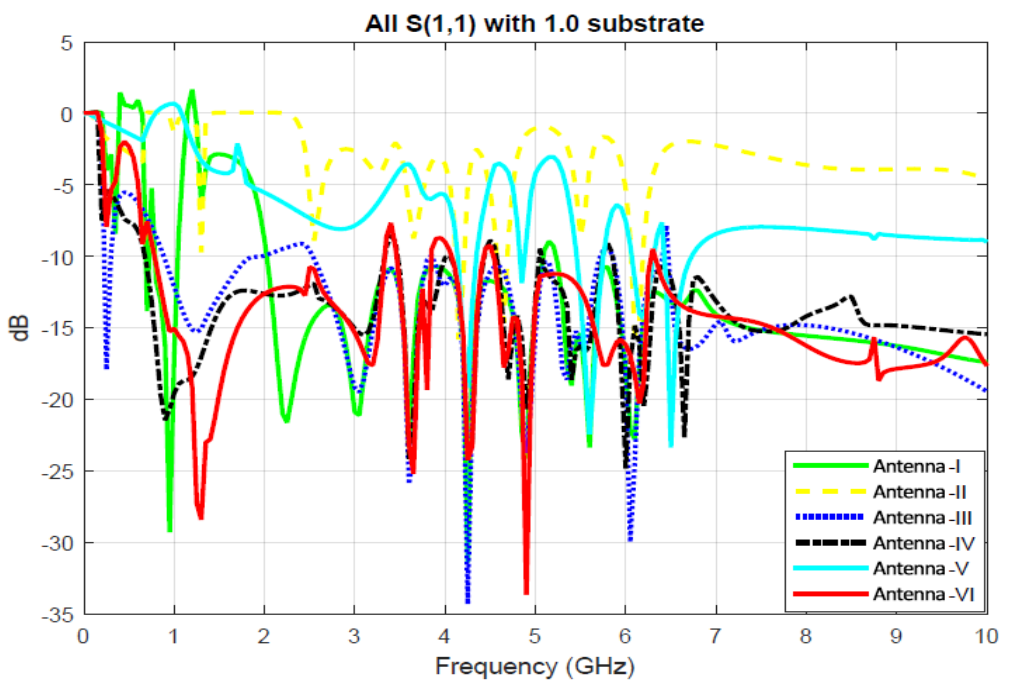

(b)

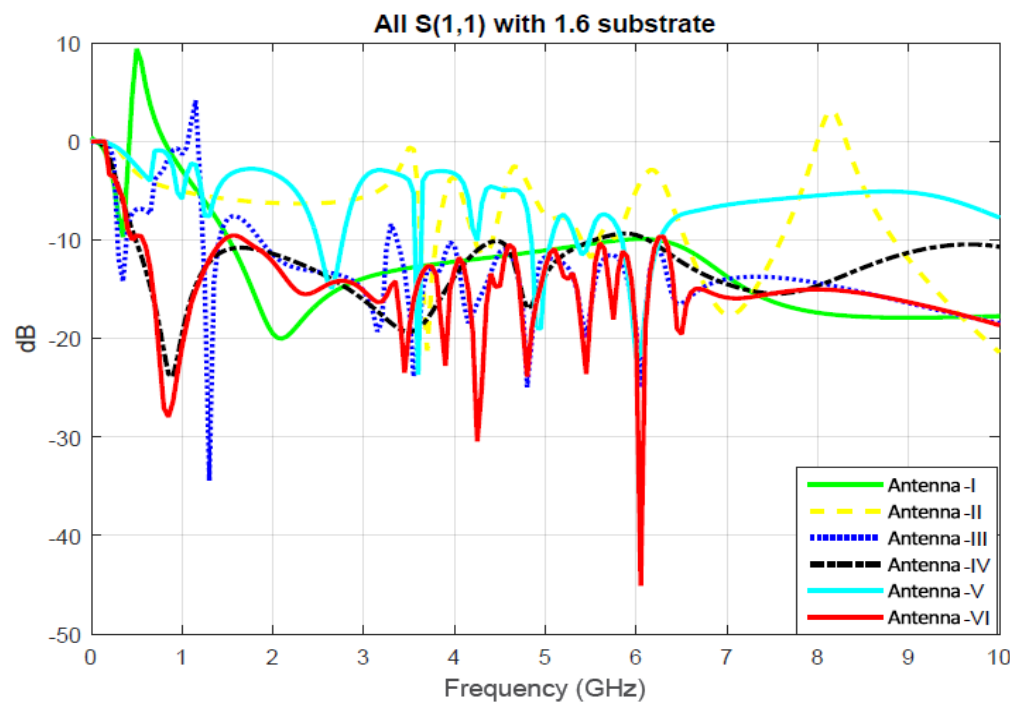

(c)

Figure 3. $\mathrm{S} 11$ graphs of simulated antennas. Substrate thickness is (a) $0.8 \mathrm{~mm}$ (b) $1.0 \mathrm{~mm}$ (c) $1.6 \mathrm{~mm}$ 
Figure 4 shows the last design of antenna in simulation and fabricated antenna results of S11 value. Fabricated antenna bandwidth starts from the $0.45 \mathrm{GHz}$ and it works frequency independently. It means that, better and more detailed information. The proposed self-complementary antenna is designed and it has homogeneous current distribution and it can be shown in Figure 5. Fabricated antenna is shown in Figure 6.

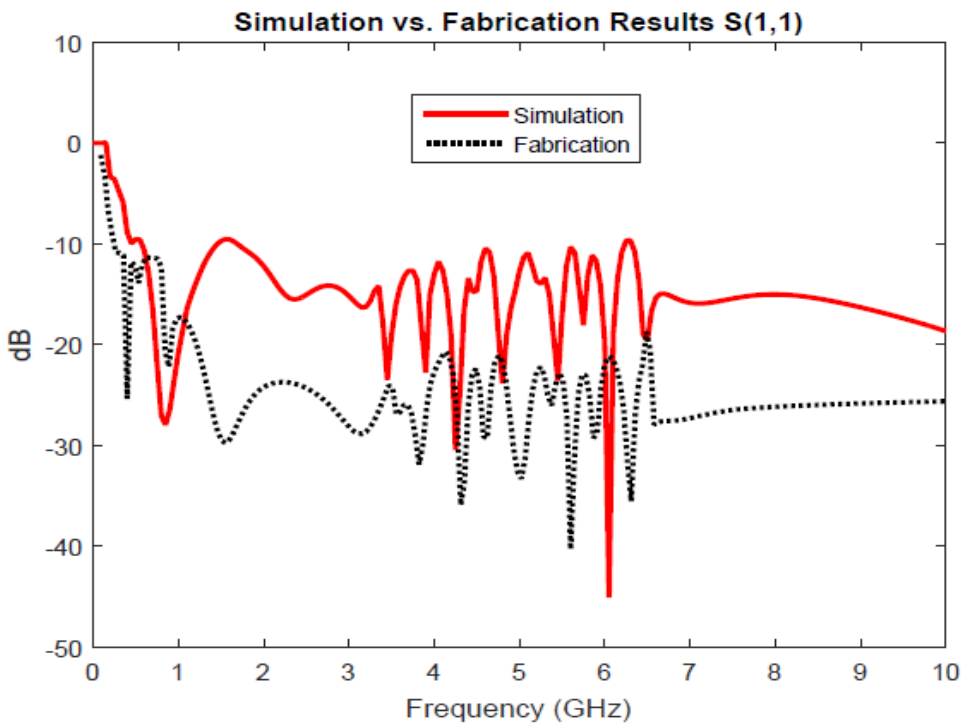

Figure 4. Measured and simulated diagrams of S11 parameter for proposed antenna

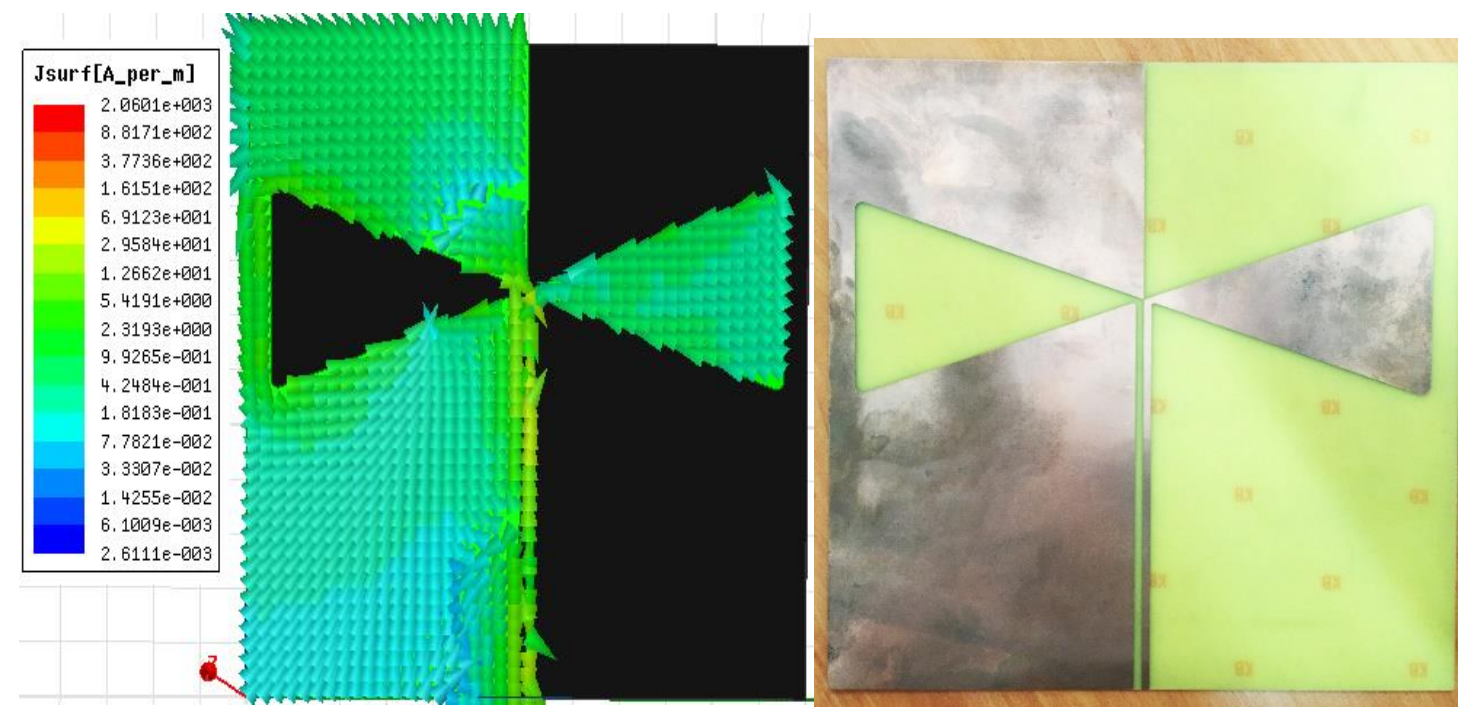

Figure 5. Demonstration of current distribution

Figure 6. Photograph of the fabricated selfcomplementary bow-tie antenna

Figure 7 shows the radiation pattern of the last version of the proposed antenna in different $\varphi$ and $\theta$ angles in $0.8 \mathrm{GHz}$ frequency. 3D graphics antenna gain value is observed in Figure 8. 
Karamzadeh et al. / Anadolu Univ. J. of Sci. and Technology A-Appl. Sci. and Eng. 18 (1) - 2017

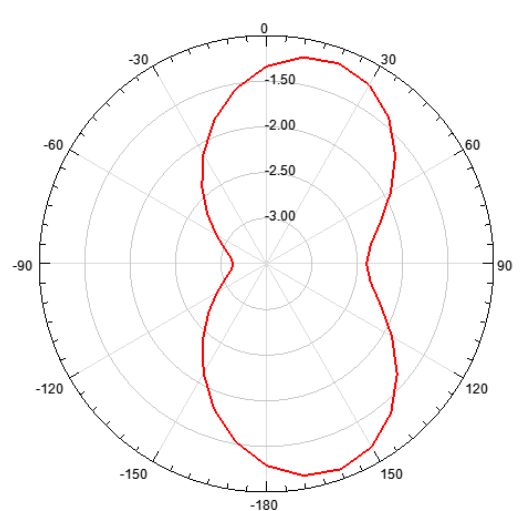

(a)

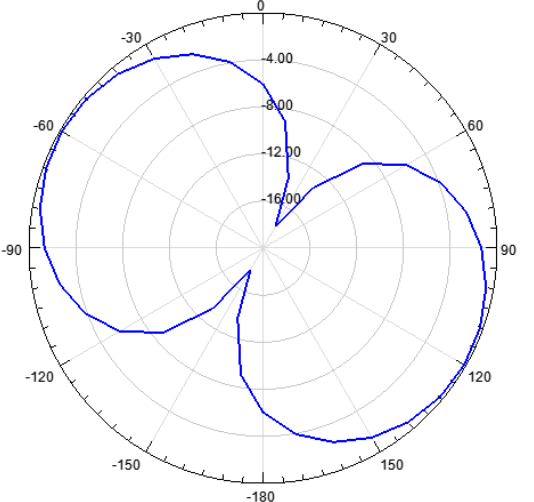

(c)

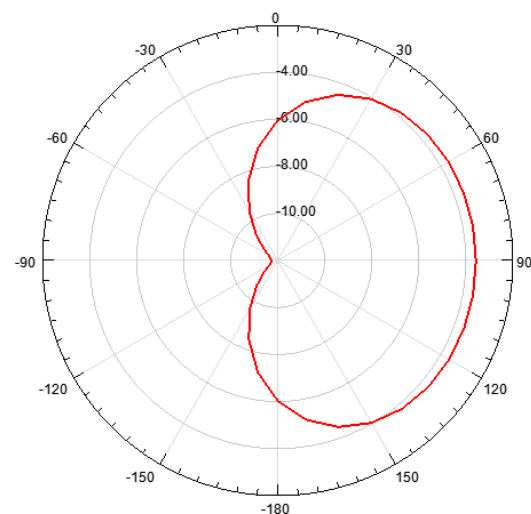

(b)

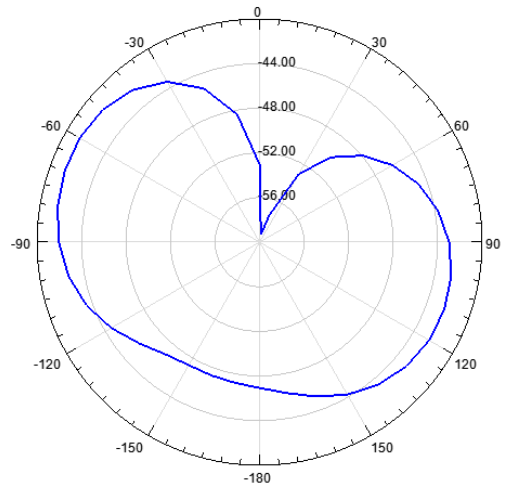

(d)

Figure 7. Radiation pattern of proposed antenna at (a) $\varphi=0^{\circ}$ (b) $\varphi=90^{\circ}$ (c) $\theta=0^{\circ}$ (d) $\theta=90^{\circ}$ at $0.8 \mathrm{GHz}$ frequency
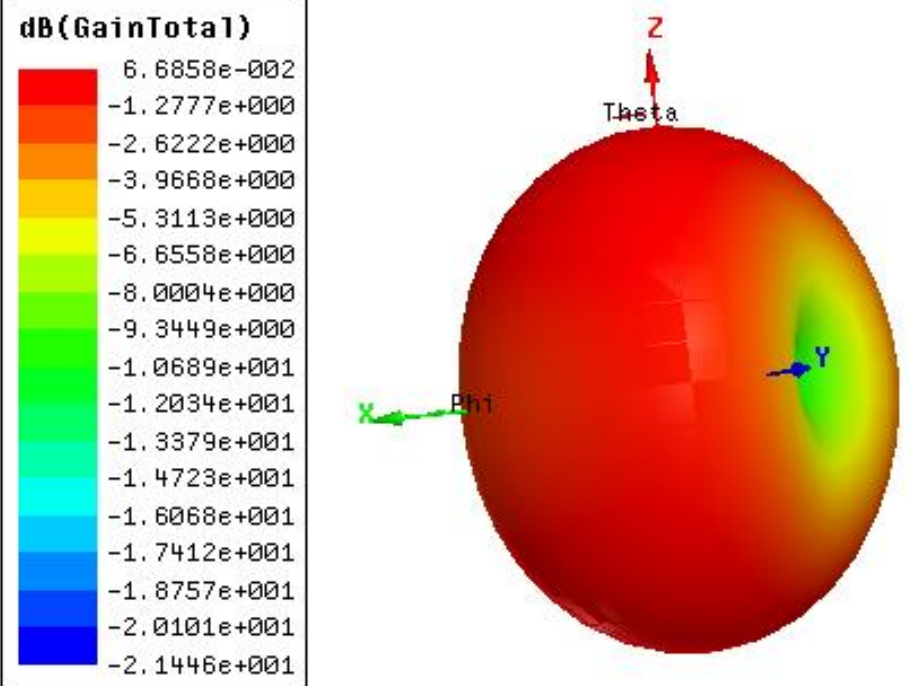

Figure 8. 3D antenna gain plot 


\section{CONCLUSION}

Antenna is the most important part of GPR systems and improvements of antenna designing will ensure better results in GPR applications. Bow-Tie antenna has several advantages for this systems and optimization studies of this antenna type provide better results for GPR applications. As a result, the size of $240 \times 240 \times 1.6 \mathrm{~mm}^{3}$ was observed that the optimum dimensions of designed antenna. FR 4 epoxy substrate whose relative permittivity is 4.4 is used for this antenna and its thickness is selected as 1.6 $\mathrm{mm}$. According to other epoxy substrates FR4 is low cost and this property is very good reason to choose this substrate. Lx and Ly values are $110 \mathrm{~mm}$ and $72.62 \mathrm{~mm}$. The best results of simulated antenna are obtained with these dimensions of the triangle feature. The angles $\alpha$ and $\theta$ are $38^{\circ}$ and $71^{\circ}$ degrees, and they give the best results. With these results; s11 values, current distribution and radiation pattern graphical representations, gain values show that these results are the best for designed antenna. Thanks to designed this self-complementary bow-tie antenna, more detailed and reliable information about an object in the deep could be obtained.

\section{REFERENCES}

[1] Skolnik MI. Radar Handbook, McGraw Hill, New York Chiago San Francisco London Madrid Mexico City Milan New Delhi San Juan Seoul Singapore Sydney Toronto, 2008.

[2] Daniels JJ. Ground Penetrating Radar Fundamentals, Department of Geological Sciences the Ohio State University, 2000.

[3] Bee TH. Detection Leakages in Underground Buried Pipe, Department of Mechanical Engineering National University in Singapore, 2009-2010

[4] Karamzadeh S, Karta M. Detection Improvement of Hidden Human's Respiratory Using Remote measurement methods with UWB Radar, International Conference on Telecommunication and Remote Sensing, Netherland, pp.(104- 108), July 2013.

[5] Karamzadeh S, Kartal M, Kent, S, Ashtiyani AA. Optimal Signal Processing Method in UWB Radar for Hidden Human Detection. In EUSAR 2014; 10th European Conference on Synthetic Aperture Radar; Proceedings of (pp. 1-3). VDE. (2014, June).

[6] Karamzadeh S, Kartal M. UWB Radar In Hidden Human Detection, International Journal of Electronics, Mechanical and Mechatronics Engineering Vol.3 Num 2 pp.(579-583), 2014.

[7] ORFEUS, Test site selection criteria for the Bore-Head Radar test programmer, Work Package: 2100 Document D3a, May 2007

[8] D. Daniels, Ground Penetrating Radar, London, UK: The Institution of Electrical Engineers, 2004.

[9] Karamzadeh S, Kartal, M. Circularly polarized $1 \times 4$ square slot array antenna by utilizing compacted modified butler matrix and branch line coupler, International Journal of RF and Microwave Computer Aided Engineering, 2015

[10] Karamzadeh S. Kartal M. Circularly polarised MIMO tapered slot antenna array for C band application. Electronics Letters 51(18), 2015.

[11] Karamzadeh S, Kartal M, Rafii V, Virdee BS. Modified circularly polarised beam steering array antenna by utilized broadband coupler and $4 \times 4$ butler matrix. IET Microwaves Antennas \& Propagation 9(9) April 2015. 
Karamzadeh et al. / Anadolu Univ. J. of Sci. and Technology A-Appl. Sci. and Eng. 18 (1) - 2017

[12] Balanis C. Antenna Theory: Analysis and Design. Wiley, 2012.

[13] Mushiake Y. Self-Complementary Antennas, IEEE Antennas and Propagation Magazine, vol. 34, no. 6, December 1992, pp.23-29.

[14] Mushiake Y, "A Report on Japanese Development of Antennas: From the Yagi-Uda Antenna to Self-Complementary Antennas, IEEE Antennas and Propagation Magazine, vol. 46, no. 4, August 2004, pp. $47-60$

[15] Azadegan R and Sarabandi K. Bandwidth enhancement of miniaturized slot antennas using folded, complementary, and self-complementary realizations. IEEE Trans. Antennas Propag., vol. 55, no. 9, pp. 2435-2444, Sep. 2007. 